\title{
The mechanisms responsible for the radiosensitizing effects of sorafenib on colon cancer cells
}

\author{
EUN HO KIM ${ }^{1}$, MI-SOOK KIM ${ }^{2}$ and WON-GYUN JUNG ${ }^{1}$ \\ ${ }^{1}$ Division of Heavy Ion Clinical Research and ${ }^{2}$ Department of Radiation Oncology, \\ Korea Institute of Radiological and Medical Sciences, Seoul 139-706, Republic of Korea
}

Received May 27, 2014; Accepted August 18, 2014

DOI: $10.3892 /$ or.2014.3497

\begin{abstract}
Colorectal cancer is one of the most common malignancies in the world, and is generally treated more effectively by chemoradiotherapy rather than radiotherapy or chemotherapy alone. Targeted radiosensitizers are often used in order to enhance the radiosensitivity of tumor cells. The aim of the present study was to identify the mechanism of radiosensitization by sorafenib in colorectal cancer. Three human colorectal adenocarcinoma cell lines (HCT116, HT29 and SW480) were treated with sorafenib alone or radiation followed by sorafenib. In vitro tests were performed using colony forming assays, FACS analysis, immunohistochemistry, tumor cell motility assays, invasion assays and endothelial tube formation assays. Sorafenib enhanced the anti-proliferative effects of radiation, reducing colony formation, increasing $\mathrm{G} 2 / \mathrm{M}$ arrest and enhancing radiation-induced apoptosis by reactive oxygen species. Sorafenib also inhibited the repair of radiation-induced DNA damage by blocking the activation of DNA-dependent protein kinase. Combination treatment significantly inhibited tumor cell migration, tumor cell invasion and vascular endothelial growth factor-mediated angiogenesis in vitro. Taken together, our results provide a scientific rationale for the use of sorafenib with radiotherapy in colon cancer and suggest a clinical utility for this approach.
\end{abstract}

\section{Introduction}

Colon cancer, the second leading cause of cancer-related mortality in the USA, has become a common malignancy in Asia with recent changes in diet (1). Radiotherapy is a standard therapy in the adjuvant treatment of resected colon and rectum cancers (2), and its combination with chemotherapy has been shown to reduce local failure and distant metastasis further, thereby improving the outcome of treatment $(3,4)$. One potential chemotherapeutic agent for this, sorafenib (Nexavar,

Correspondence to: Dr Mi-Sook Kim, Department of Radiation Oncology, Korea Institute of Radiological and Medical Sciences, 215-4 Gongneung-Dong, Nowon-Ku, Seoul 139-706, Republic of Korea E-mail: eh140149@kirams.re.kr

Key words: angiogenesis, colon cancer cells, radiosensitivity, DNA damage, $\gamma$-radiation
BAY43-9006), is an oral multikinase inhibitor that blocks tumor cell proliferation and angiogenesis, and induces tumor cell apoptosis by inhibiting serine/threonine kinases (c-RAF and mutant and wild-type BRAF) as well as the receptor tyrosine kinases vascular endothelial growth factor receptor 2 and 3 (VEGFR2 and VEGFR3), platelet-derived growth factor receptor $\beta$, FLT3 and c-KIT (5). Sorafenib is currently used in clinics to treat patients with advanced renal cell carcinoma, hepatocellular carcinoma and thyroid cancer (6-10). Moreover, preliminary data from a series of studies in which sorafenib was used in combination with a variety of anticancer agents for various solid tumors has been published (11).

In the present study, we investigated the mechanism by which sorafenib enhances radiation-induced antitumor and anti-angiogenesis effects in colorectal cancer cells. Collectively, our results showed that sorafenib can be successfully combined with a radiation regimen to potentiate its antitumor and anti-angiogenesis activities. Our study provides a scientific rationale to evaluate this combination strategy in clinical trials.

\section{Materials and methods}

Antibodies and chemicals. Anti-cyclin B, anti-cyclin A, anti- $\beta$-actin and anti-matrix metalloproteinase-9 (MMP-9) were purchased from Santa Cruz Biotechnology. Anti-cleaved poly(ADP-ribose) polymerase-1 (PARP1) antibody, anticleaved caspase 3 , anti-pDNA-dependent protein kinase (DNA-PK; S2059), anti-PERK and anti-pAkt were purchased from Cell Signaling Technology (Danvers, MA, USA), and anti-phosphorylated $\mathrm{H} 2 \mathrm{AX}(\gamma \mathrm{H} 2 \mathrm{AX})$ was obtained from Millipore (Billerica, MA, USA). Sorafenib was purchased from Selleckchem. For in vitro experiments, sorafenib was dissolved in dimethyl sulfoxide to make a $16 \mathrm{mmol} / \mathrm{l}$ stock solution and was stored at $4^{\circ} \mathrm{C}$.

Cell culture. The human colorectal cancer cell lines HT29, HCT116 and SW480 were grown in RPMI-1640 medium supplemented with $10 \%$ fetal bovine serum (FBS), glutamine, HEPES and antibiotics at $37^{\circ} \mathrm{C}$ in a $5 \% \mathrm{CO}_{2}$ humidified incubator. Human umbilical vein endothelial cells (HUVECs) were maintained in endothelial cell basal medium (EGM-2; Cambrex) containing EGM-2 SingleQuot growth supplements (Cambrex) and maintained for no more than 8 culture passages. 
Irradiation. Cells were plated in $60-\mathrm{mm}$ diameter dishes and incubated at $37^{\circ} \mathrm{C}$ under humidified conditions and $5 \% \mathrm{CO}_{2}$ until they reached 70-80\% confluence. Cells were irradiated with a ${ }^{137} \mathrm{Cs} \gamma$-ray source (Atomic Energy of Canada, Ltd., Ontario, Canada) at a dose rate of $3.81 \mathrm{~Gy} / \mathrm{min}$.

Colony-forming assay. Sorafenib was added to cells $1 \mathrm{~h}$ after radiation exposure to a final concentration of $16 \mu \mathrm{mol} / 1$, and the cells were then incubated for $72 \mathrm{~h}$. After 14-20 days, colonies were stained with $0.4 \%$ crystal violet (Sigma, St. Louis, MO, USA). The plating efficiency (PE) represents the percentage of seeded cells that grew into colonies under the specific culture conditions of a given cell line. The survival fraction, expressed as a function of irradiation, was calculated as follows: Survival fraction $=$ colonies counted $/($ cells seeded $\times \mathrm{PE} / 100)$. PEs of HT29, HCT116 and SW480 were $0.52 \pm 0.18,0.50 \pm 0.15$, and $0.48 \pm 0.10$, respectively. To evaluate the radiosensitizing effects of sorafenib, the ratio for radiation alone and radiation plus sorafenib was calculated as the dose (Gy) for radiation alone divided by the dose for radiation plus sorafenib at a surviving fraction of $50 \% 50 \%$ of cells.

Flow cytometry. Cells were cultured, harvested at the indicated times, stained with propidium iodide (PI; $1 \mu \mathrm{g} / \mathrm{ml}$, Sigma), according to the manufacturer's protocol, and then analyzed using a FACScan flow cytometer (Becton Dickinson, Franklin Lakes, NJ, USA). A minimum of 10,000 cells was counted for each sample, and data analysis was performed with the use of CellQuest software (BD Biosciences).

Detection of apoptotic cells by Annexin V staining. After the exposure to radiation, sorafenib was added to the cells, which were then incubated for a further $48 \mathrm{~h}$. Cells were washed with ice-cold phosphate-buffered saline (PBS), trypsinized and re-suspended in $1 \mathrm{X}$ binding buffer $[10 \mathrm{~mm}$ HEPES/NaOH (pH 7.4), $140 \mathrm{~mm} \mathrm{NaCl}$ and $\left.2.5 \mathrm{~mm} \mathrm{CaCl}_{2}\right]$ at $1 \times 10^{6}$ cells $/ \mathrm{ml}$. Aliquots $(100 \mu \mathrm{l})$ of cell solution were mixed with $5 \mu 1$ Annexin V FITC (Pharmingen) and $10 \mu 1$ PI stock solution $(50 \mu \mathrm{g} / \mathrm{ml}$ in PBS) by gentle vortexing, followed by $15 \mathrm{~min}$ of incubation at room temperature in the dark. Buffer $(400 \mu 1,1 \mathrm{X})$ was added to each sample and analyzed on a FACScan flow cytometer (Becton Dickinson). A minimum of 10,000 cells was counted for each sample, and data analysis was performed using CellQuest software (BD Biosciences).

Fluorescent measurement of intracellular reactive oxygen species. The fluorescent probe 2',7'-dichlorofluorescin diacetate (DCFH-DA) was used for the assessment of intracellular reactive oxygen species (ROS). For fluorocytometrical analysis, cells were plated in $60-\mathrm{mm}$ diameter dishes $\left(1 \times 10^{5}\right.$ cells/dish) and loaded for $30 \mathrm{~min}$ at room temperature with $10 \mu \mathrm{M}$ DCFH-DA in $5 \mathrm{ml}$ PBS. Unincorporated DCFH-DA was removed with 2 washes in PBS. DCFH-DA-loaded cells were treated with sorafenib or radiation alone, or in combination. Fluorescence was measured using a flow cytometer (Becton Dickinson).

Immunohistochemistry. Immunohistochemistry was performed to determine the nuclear distribution of $\gamma-\mathrm{H} 2 \mathrm{AX}$ in individual cells. Cells were grown on chambered slides 1 day prior to irradiation or sorafenib treatment. After radiation exposure, sorafenib was added to the cells, and the cells were treated for various lengths of time. All treatments were performed while cells remained attached to the slides, followed by fixing with $4 \%$ paraformaldehyde and permeabilization with $0.2 \%$ Triton $\mathrm{X}-100$ in PBS. Detection was performed after blocking the slides in $10 \% \mathrm{FBS} / 1 \%$ bovine serum albumin for $1 \mathrm{~h}$ at a 1:1,000 dilution of FITC-labeled mouse monoclonal antibody against $\gamma-\mathrm{H} 2 \mathrm{AX}$ (Millipore) in the background-reducing antibody diluent (DAKO plus S3022; Millipore).

Western blotting. Following irradiation, sorafenib was added to the colon cancer cells, which were then incubated for 1 or $24 \mathrm{~h}$. The cells were then lysed with RIPA buffer. Proteins were separated by sodium-polyacrylamide gel electrophoresis and transferred to nitrocellulose membranes. The membranes were blocked with $1 \%(\mathrm{v} / \mathrm{v})$ non-fat dried milk in Tris-buffered saline with $0.05 \%$ Tween-20 and incubated with the required antibodies. Primary antibodies were used at a 1:1,000 dilution and secondary antibodies at a 1:5,000 dilution. Immunoreactive protein bands were visualized by enhanced chemiluminescence (Amersham Biosciences) and scanned.

Tumor cell motility assay. The cell motility assay was conducted in 6-well plates. A fine scratch in the form of a groove was made using a sterile pipette tip in a layer of cells at $\sim 90 \%$ confluency. Cells were then treated with sorafenib, radiation or a combination of both. The migration of cells was monitored using a Nikon Eclipse Ti microscope with a DS-Fi1 camera.

Invasion assay. The invasive ability was measured in vitro using Transwell chambers, according to the manufacturer's protocol. Briefly, cells were seeded onto the membrane of the upper chamber of the Transwell at a concentration of $4 \times 10^{5}$ cells $/ \mathrm{ml}$ in $150 \mu \mathrm{l}$ of RPMI medium and were left untreated or treated with the indicated doses of sorafenib, radiation, or a combination of both for $24 \mathrm{~h}$. The medium in the upper chamber was serum-free, while the medium in the lower chamber contained $10 \%$ FBS as a source of chemo-attractants. Cells that passed through the Matrigel-coated membrane were stained with Cell Stain Solution containing crystal violet supplied in the Transwell invasion assay (Chemicon, Millipore) and images were captured after $24 \mathrm{~h}$ of incubation.

Matrigel in vitro endothelial tube formation assay. Endothelial cell tube formation was assessed using Matrigel-coated chamber slides as previously described (12). The results of each assay were photographed (Nikon Eclipse Ti microscope with DS-Fil camera) at $\mathrm{x} 40$ magnification. Tube formation was quantified by counting the number of connected cells in randomly selected fields at $\mathrm{x} 400$ magnification with a microscope, and dividing that number by the total number of cells in the same field.

Statistical analysis. All data were plotted as the mean \pm standard error of the mean (SEM). Results of colony forming assays were analyzed using a paired t-test with SPSS 18.0 software (SPSS, Chicago, IL, USA). All other data were analyzed by parametric repeated measure one-way analysis of variance (ANOVA), 

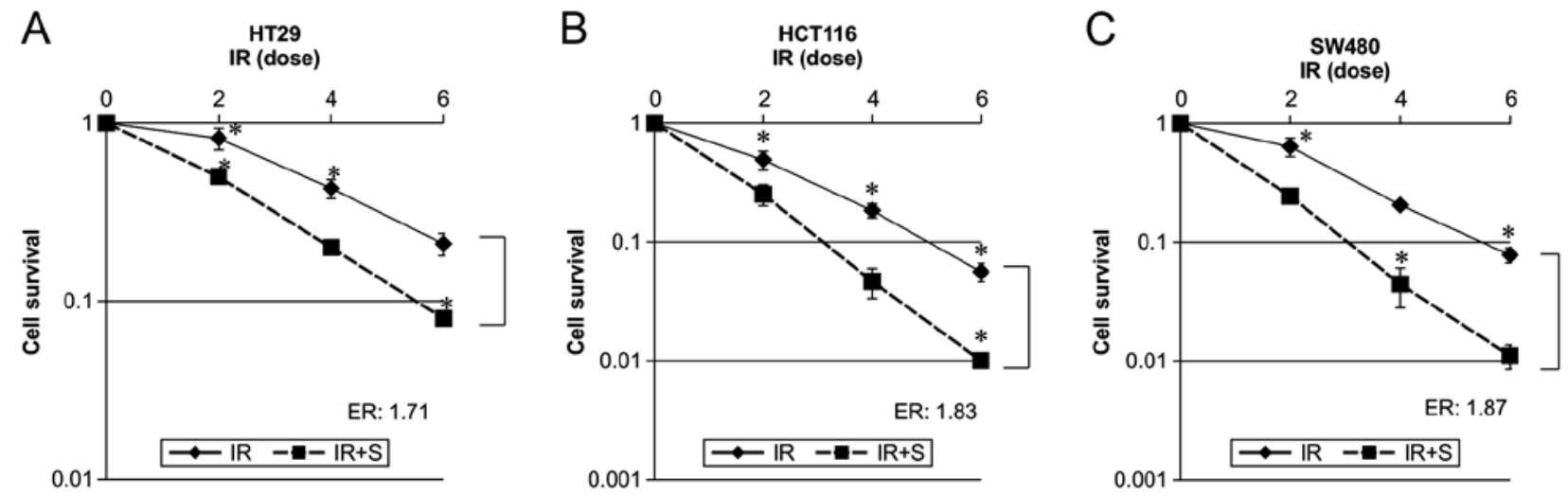

Figure 1. Radiosensitizing effects of sorafenib on colon cancer cells. Radiosensitivity of HT29, HCT116 and SW480 cell lines with and without post-radiation sorafenib ( $16 \mu \mathrm{mol} / \mathrm{l})$ was measured by colony forming assay. Asterisks (") indicate values that are statistically significant in comparison to radiation-treated cells. Values represent means of three experiments $\pm \mathrm{SE}$; ${ }^{*} \mathrm{P}<0.05$. ER $=$ enhancement ratio.

followed by Tukey's honestly significant difference test (SPSS 18.0). Statistical significance was set at $\mathrm{P}<0.05$.

\section{Results}

Cooperative effect of radiation and sorafenib on colon cancer cell proliferation. In order to evaluate the effects of sorafenib on radiation-induced cytotoxicity, a clonogenic survival assay was performed using HT29, HCT116 and SW480 cells. We used a sorafenib concentration resulting in a $25 \%$ growth inhibition after a 48-h exposure in each experiment (data not shown), and added this drug post-radiation as this was previously shown to be more efficacious than pre-radiation treatment (13). Dose-response curves of the 3 colon cancer cell lines irradiated in the presence or absence of sorafenib are shown in Fig. 1. Combined radiation and sorafenib treatment was more effective than radiation alone in all cases. The effect of sorafenib on radiation-mediated cell killing was expressed as an enhancement ratio $\left(D_{50}\right)$.

Sorafenib enhances radiation-induced apoptosis. To investigate whether sorafenib and radiation induce apoptosis, we assessed early apoptosis by Annexin V and PI staining. In colon cancer cell lines, combined 48-h sorafenib treatment and radiation exposure significantly increased the proportion of cells in early apoptosis in all 3 colon cancer cell lines (Fig. 2A). We next investigated whether sorafenib-enhanced radiation cytotoxicity resulted from the increased activation of caspase, resulting in enhanced apoptotic cell death. We observed increased activation of caspase- 3 and increased PARP cleavage in response to combined radiation and sorafenib treatment compared to sorafenib alone (Fig. 2B). We also investigated the relationship between ROS production and enhancement of radiation-induced apoptosis by sorafenib. The production of ROS was synergistically induced by the combined treatment of sorafenib and radiation in colon cancer cell lines (Fig. 2C), indicating that ROS generated by the combined treatment increases intracellular caspase signaling and, thus, apoptosis.

Effects of sorafenib and radiation on the cell cycle. We next investigated whether combined sorafenib/radiation-induced cytotoxicity resulted from differences in cell cycle regulation by analyzing cell cycle progression through flow cytometry. Sorafenib treatment combined with radiation significantly increased the proportion of cells in $\mathrm{G} 2$ to $\mathrm{M}$ phase for all 3 cell lines, compared to radiation alone (Fig. 3A). We also examined the expression of cell cycle regulators after combined sorafenib and radiation treatment. Western blotting revealed that radiation alone resulted in a significant accumulation of cyclin A and cyclin B, a key cell cycle regulator involved in the $\mathrm{G} 2 / \mathrm{M}$ transition, whereas sorafenib alone and combined treatment reduced expression of cyclin A and cyclin B levels (Fig. 3B).

Influence of sorafenib on radiation-induced DNA damage and DNA repair activity. To analyze the effect of sorafenib on double-strand break (DSB) repair, the level of $\gamma \mathrm{H} 2 \mathrm{AX}$, a marker for DSB, was examined by immunofluorescence and western blotting 0,1 and $24 \mathrm{~h}$ after treatment. Prolonged expression of $\gamma \mathrm{H} 2 \mathrm{AX}$ was observed after 24-h radiation exposure in the presence of sorafenib (Fig. 4A). All 3 colon cancer cell lines treated with combined sorafenib and irradiation exhibited damaged DNA foci, which appeared $1 \mathrm{~h}$ after treatment and were still present $24 \mathrm{~h}$ after exposure (Fig 4A). In addition, cell lysates were immunoblotted with anti- $\gamma \mathrm{H} 2 \mathrm{AX}$ antibody (Fig. 4B), which confirmed the results of the immunofluorescence assay, and the levels of phosphorylated DNA-PK were slightly reduced after combined treatment (Fig. 4C).

Combination treatment significantly inhibits tumor cell motility and tumor cell invasion. We next evaluated the effects of sorafenib and radiation on the invasive and migratory capacities of colon cancer cells using Matrigel and scratch assays. In the latter, combined sorafenib and radiation treatment significantly inhibited cell migration compared with sorafenib or radiation alone (Fig. 5A and B). In the Matrigel invasion assay, combined sorafenib and radiation treatment was highly effective in inhibiting the invasive behavior of all 3 colon cancer cell lines, by 10,15 and $8 \%$ (Fig. 5C and D). We also evaluated the extent to which MMP-9 expression is altered in colon cancer cells treated with sorafenib and radiation. Notably, sorafenib suppressed the upregulation of MMP-9 caused by irradiation (Fig. 5E). 

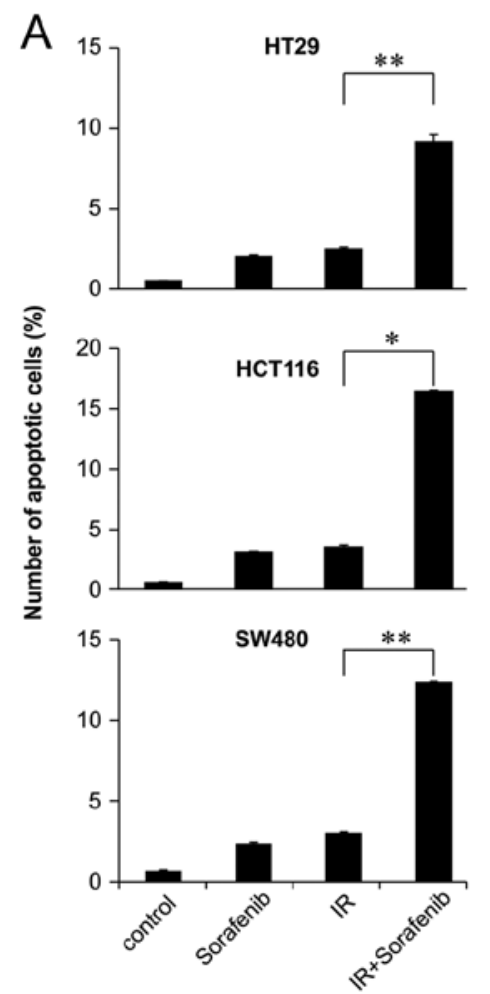

B

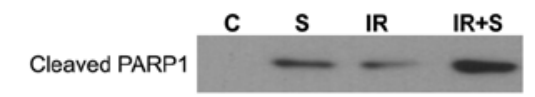

突

Cleaved Caspase-3

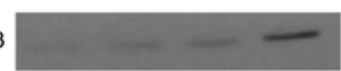

$\beta$-actin
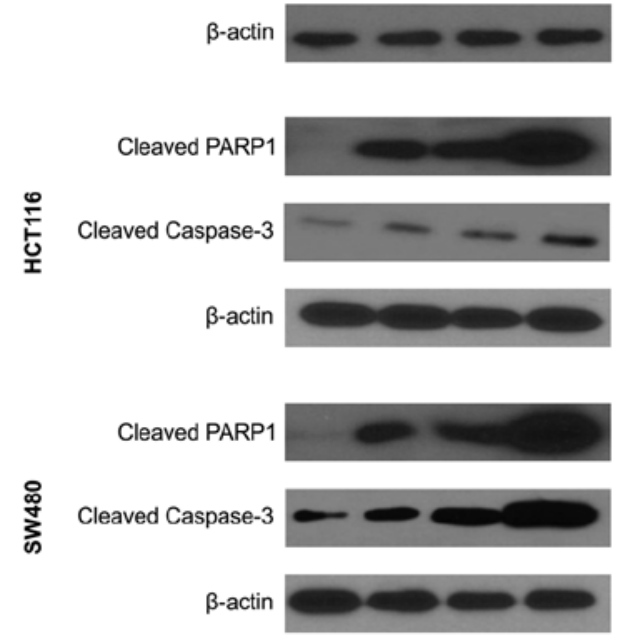
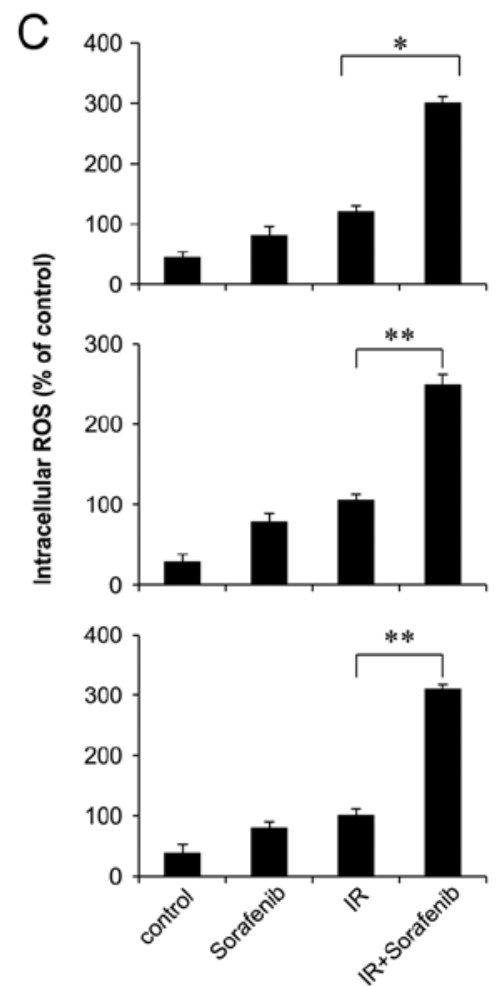

Figure 2. Effects of sorafenib and radiation on apoptosis in colon cancer cells. (A) HT29, HCT116 and SW480 cell lines were exposed to sorafenib (16 $\mu$ mol/1) and/or $5 \mathrm{~Gy}$ radiation for $48 \mathrm{~h}$ for Annexin V staining. Values represent means of three experiments $\pm \mathrm{SE}$; ${ }^{\mathrm{P}}<0.05$, "* $\mathrm{P}<0.001$. (B) Cell lysates (30 $\left.\mu \mathrm{g}\right)$ were immunoblotted (IB) with antibodies against cleaved PARP1, cleaved caspase-3 and $\beta$-actin. (C) HT29, HCT116 and SW480 cells were treated with sorafenib, radiation, or the indicated combinations, and ROS levels were determined by DCFH-DA flow cytometry. Data are expressed as \% of control and are means $\pm \mathrm{SE}$ of 3 or 4 experiments. Values represent means of three experiments $\pm \mathrm{SE} ;{ }^{*} \mathrm{P}<0.05,{ }^{* *} \mathrm{P}<0.001$.

A
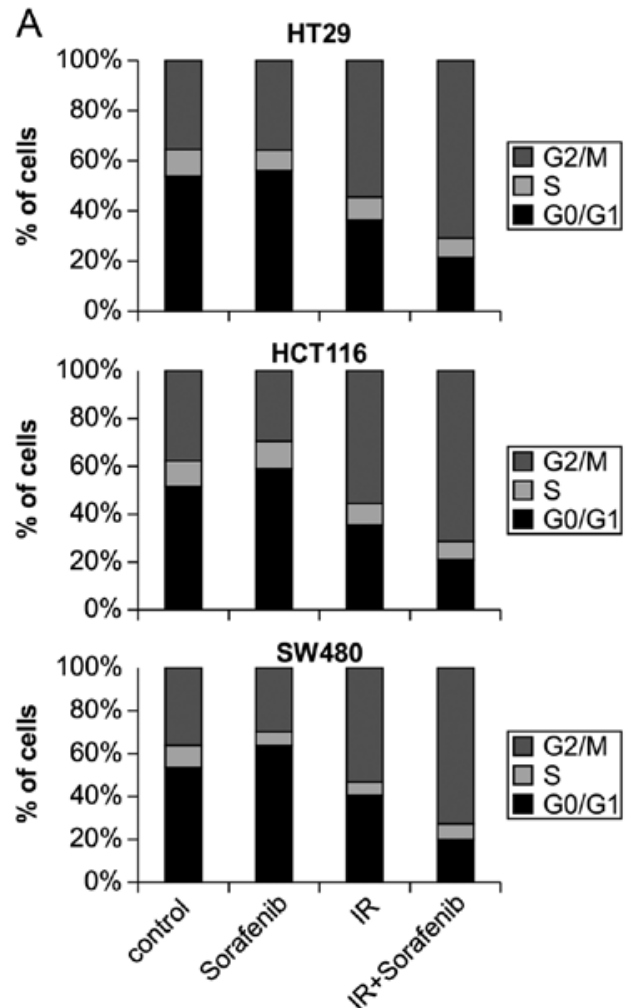

B
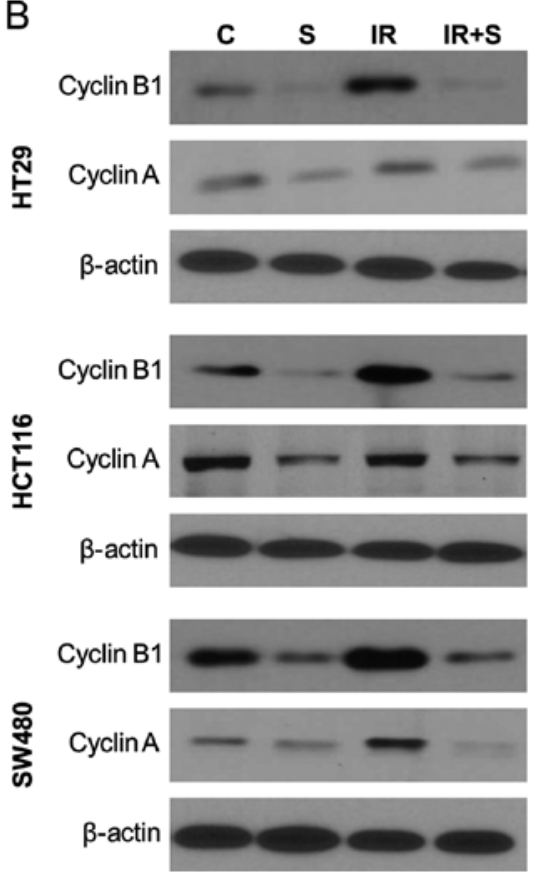

Figure 3. Sorafenib blocks cell cycle progression in irradiated cells in the G2 to M phase and alters the expression of cyclin A and cyclin B1. (A) HT29, HCT116 and SW480 cell lines were treated with sorafenib ( $16 \mu \mathrm{mol} / \mathrm{l})$ and/or $5 \mathrm{~Gy}$ radiation for $24 \mathrm{~h}$. The cell cycle distribution was analyzed quantitatively. ${ }^{*} \mathrm{P}<0.05$ vs. radiation-treated cells. (B) Cyclin A and cyclin B1 expression was analyzed by western blotting. HT29, HCT116 and SW480 cell lines were treated with radiation before the addition of sorafenib $(16 \mu \mathrm{mol} / \mathrm{l})$, and incubated for $24 \mathrm{~h}$. Equal amounts of cell lysate (30 $\mu \mathrm{g})$ were separated by electrophoresis and analyzed by western blotting for cyclin A and cyclin B1. 


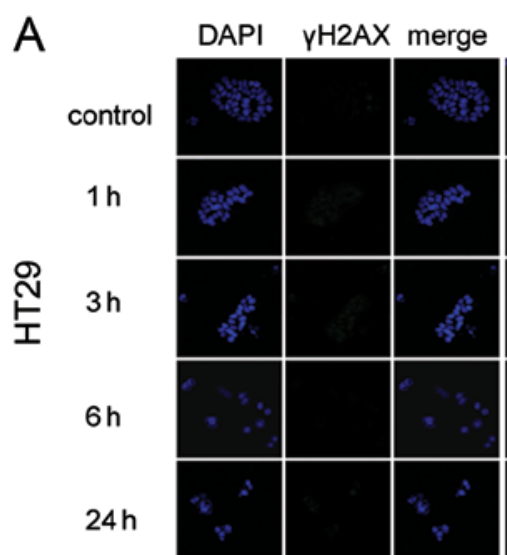

$\mathrm{S}$
DAPI yH2AX merge

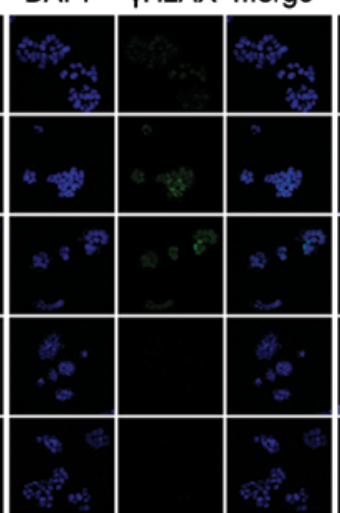

IR

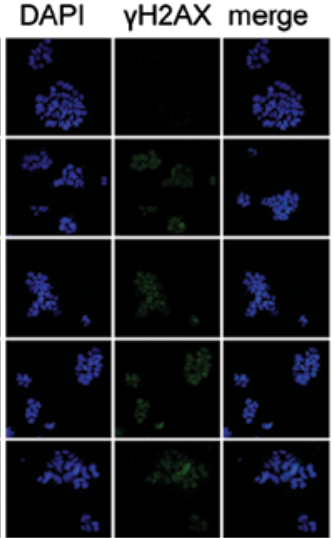

IR+S
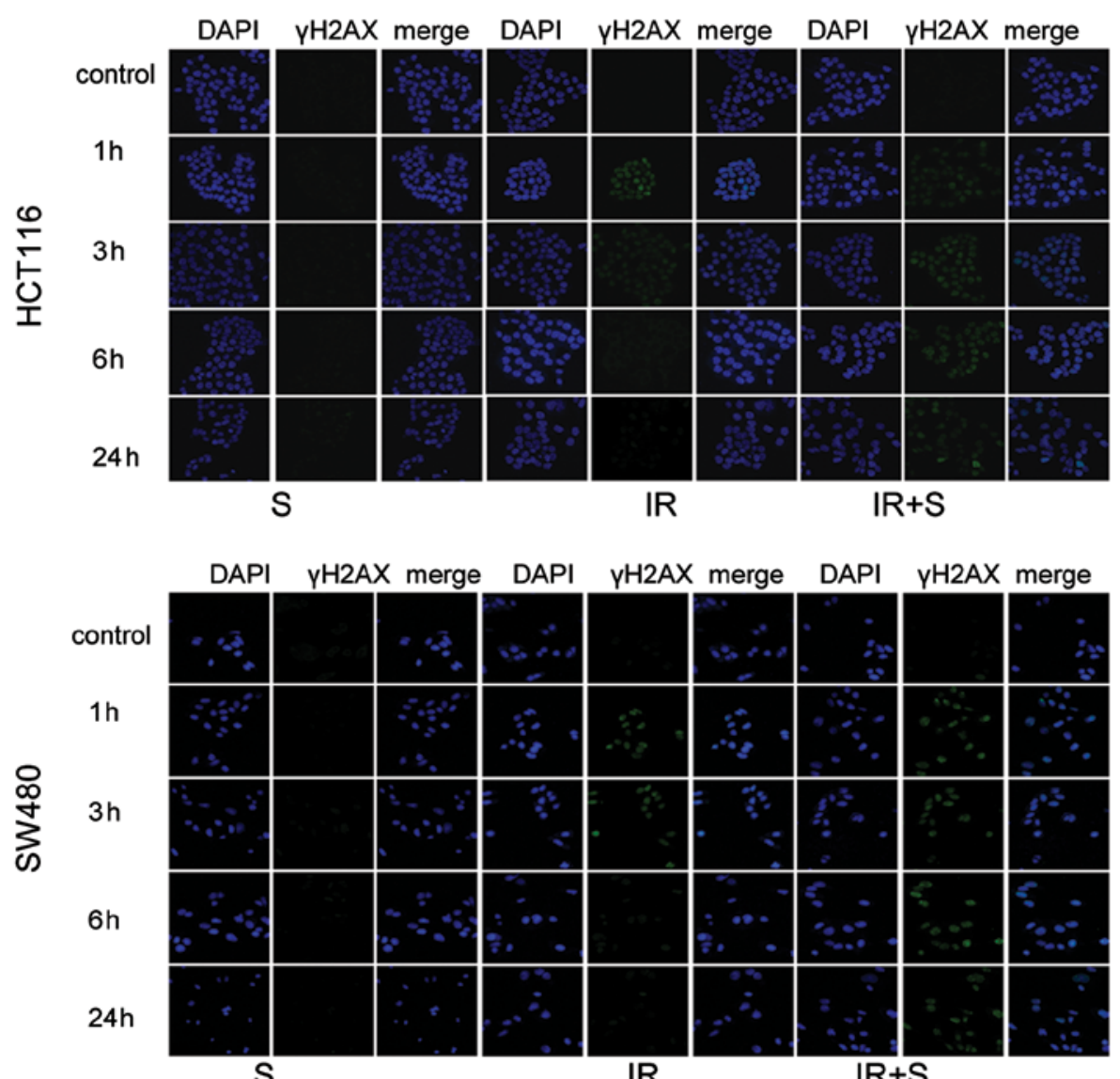

$\mathrm{s}$

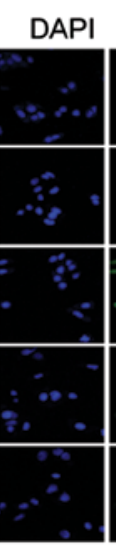

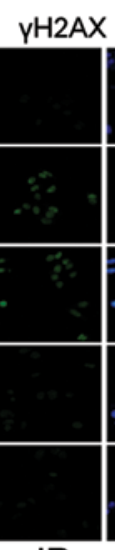

IR

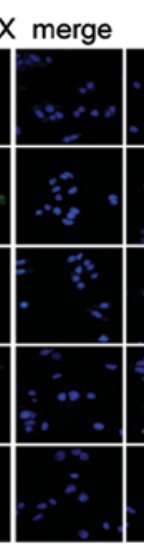

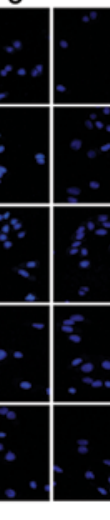

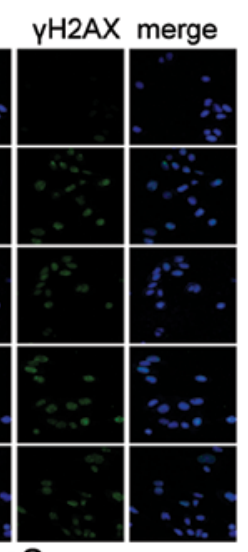

IR+S
B
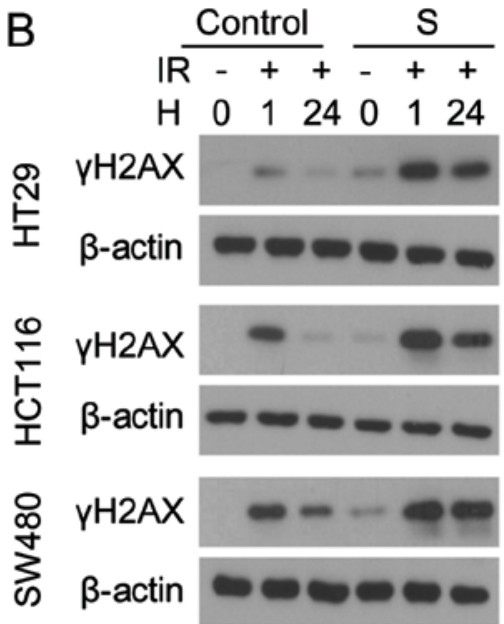

C

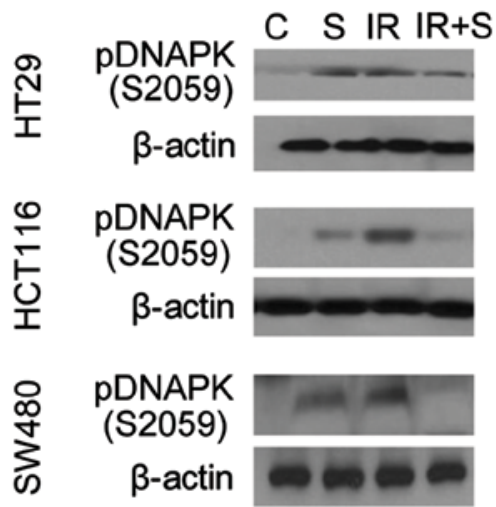

Figure 4. Effects of sorafenib on the DNA damage response in irradiated colon cancer cells. (A) Immunocytochemistry staining for H2AX phosphorylation (Ser139, green) in HT29, HCT116 and SW480 cells treated with radiation or sorafenib after various time points. (B and C) Cell lysates prepared from sorafenib, radiation, and sorafenib + radiation-treated cells were immunoblotted with the indicated antibodies.

Combination treatment significantly inhibits tumor angiogenesis. We next examined whether combined sorafenib and radiation treatment blocks angiogenesis by inhibiting VEGF. Combined sorafenib and radiation completely inhibited VEGF-mediated endothelial tube formation in HUVECs, whereas either treatment alone inhibited tube formation by only 33 and 36\%, respectively (Fig. 6A and B). VEGF predominantly mediates angiogenesis via the PI3K/Akt and MAPK signaling cascades (5), and combination treatment significantly inhibited Akt and ERK1/2 activation compared with sorafenib or radiation alone, as shown by western blotting (Fig. 6C).

\section{Discussion}

The purpose of the present study was to investigate the mechanism of radiosensitization by sorafenib on colorectal cancer cells. Several clinical trials have been initiated to evaluate the use of sorafenib in combination with a variety of anticancer agents to treat a range of tumor types. The most promising evidence of antitumor activity was observed when sorafenib was combined with interferon- $\alpha$ in renal cell carcinoma, dacarbazine in melanoma, doxorubicin in hepatocellular carcinoma and gemcitabine in ovarian cancer (14). Moreover, 


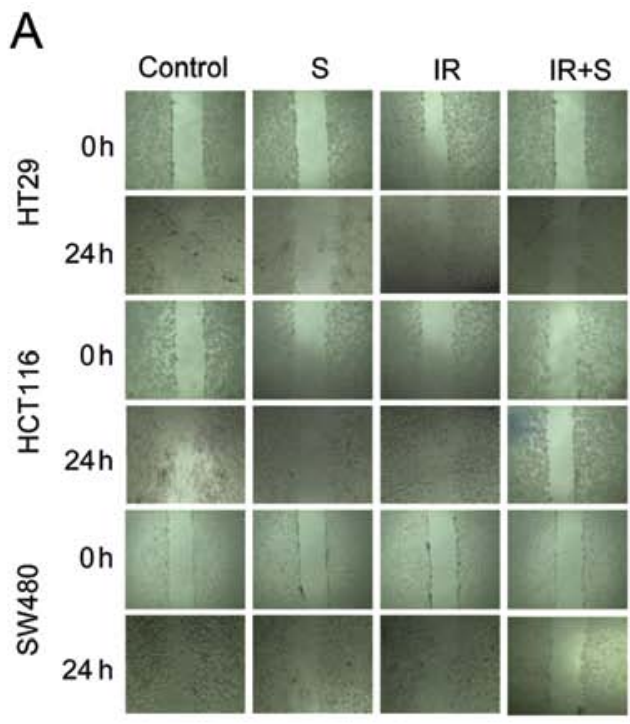

C

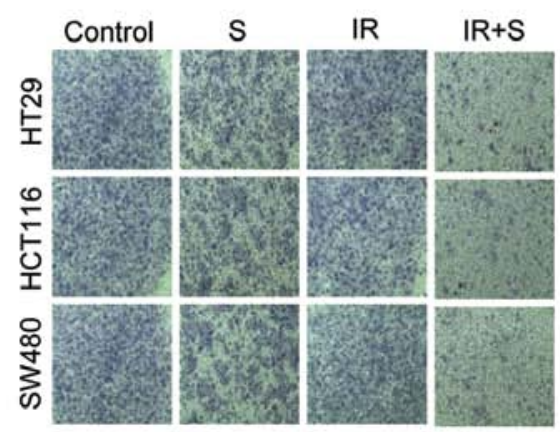

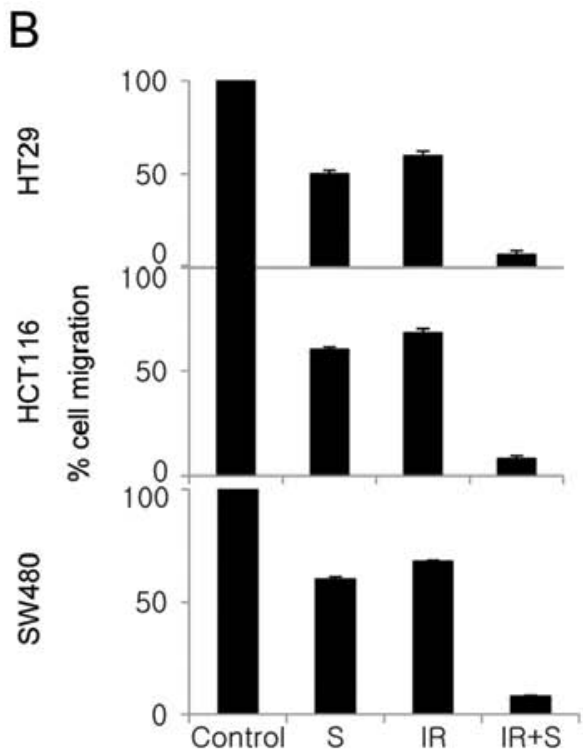

D

E

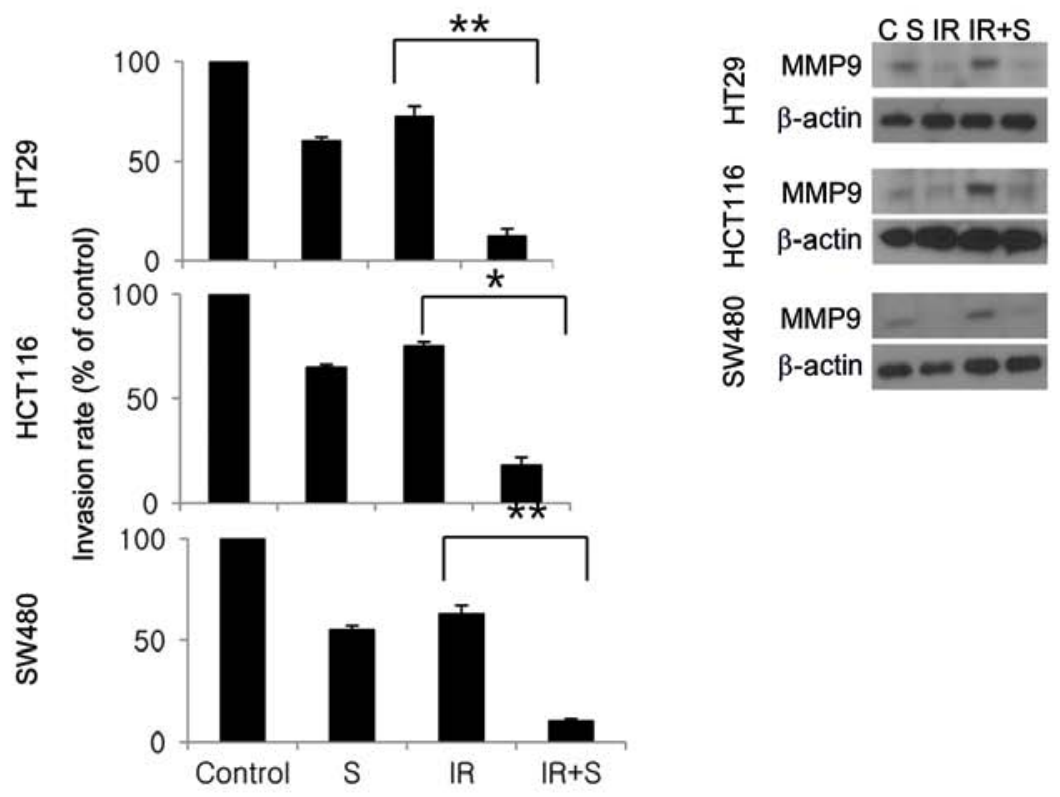

Figure 5. Effect of treatment with sorafenib and radiation on the invasion and migration of colon cancer cells. (A) Colon cancer cells were scraped with yellow tips by scratch assay, and then treated with sorafenib and radiation. After incubation for $24 \mathrm{~h}$, the number of calls that migrated across the wound was counted. (B) Each assay was photographed and distances between the migrating cell edges were quantified, and percentage of cell migration was calculated. Values represent means of three experiments $\pm \mathrm{SE} ;{ }^{*} \mathrm{P}<0.05,{ }^{* *} \mathrm{P}<0.001$. (C) Tumor cell invasion was assessed using Matrigel invasion assay. (D) The number of tumor cells that had invaded through the Matrigel was counted in 5 high power fields. Values represent means of 3 experiments $\pm \mathrm{SE}$; ${ }^{*} \mathrm{P}<0.05$, ${ }^{* *} \mathrm{P}<0.001$. $(\mathrm{E}) \mathrm{Cell}$ lysates prepared from sorafenib, radiation, and sorafenib + radiation-treated cells were immunoblotted with the indicated antibodies.

the combination of sorafenib and another targeted agent, bevacizumab, also showed promising antitumor activity in patients with ovarian cancer (15), and other clinical studies have shown that the combination of sorafenib and radiation may provide clinical benefits in other types of cancer (16-18). However, the mechanism by which radiation-enhancement occurred appeared to be somewhat more complex than predicted in previous studies. In this study, we provided a scientific rationale for the clinical application of sorafenib as a radiosensitizer in colorectal cancer. On the basis of our results, we suggest that sorafenib significantly enhances the therapeutic efficiency of radiation by inhibiting tumor cell survival, cell cycle regulation, DNA repair activity, tumor cell invasiveness, and angiogenesis in human colon adenocarcinoma cell lines. Sorafenib combined with radiation significantly reduced the clonogenic survival and enhanced the radiosensitivity of colon cancer cells by promoting apoptosis through increased ROS levels. Previous studies found that post-radiation sorafenib treatment resulted in greater radiosensitization than pre-radiation treatment (13), therefore we employed this schedule in our study. When sorafenib was administered after irradiation, the cells failed to complete mitosis owing to a block in the $\mathrm{G} 2$ to $\mathrm{M}$ phase transition, which was possibly mediated by the downregulation of cyclin B1. 
A
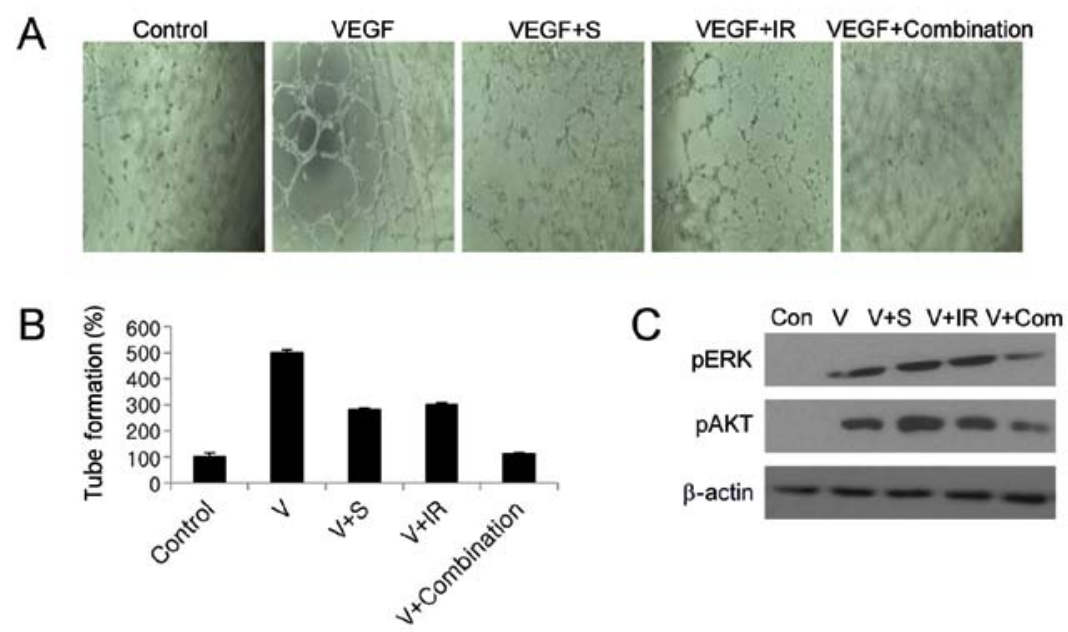

Figure 6. Effect of treatment with sorafenib and radiation on the angiogenic behavior of endothelial cells. (A) Representative photomicrographs of in vitro tube formation for control, VEGF, VEGF + sorafenib, VEGF + radiation and VEGF + combination treatment experiments. (B) Quantitative data for tube formation expressed as angiogenic score \pm SE from 3 independent experiments. (C) Endothelial cells were treated with sorafenib and/or 5 Gy radiation for $1 \mathrm{~h}$ and then with VEGF for $30 \mathrm{~min}$. Whole cell lysates from each group were immunoblotted with the indicated antibodies.

Radiotherapy is one of the major therapeutic strategies for cancer treatment and results in DNA damage, including DSBs, which in turn initiates a variety of signaling events in cancer cells (19). DSBs lead to the phosphorylation of H2AX, and we and others have used this as a marker for the cellular response to radiation-induced DNA damage (20-22). Our results showed that the combined treatment of sorafenib and radiation delayed the clearance of $\gamma \mathrm{H} 2 \mathrm{AX}$, suggesting that sorafenib prevents DNA repair and hence increases radiosensitivity. Homologous recombination and non-homologous end-joining (NHEJ) are two major pathways for the repair of DNA DSBs. NHEJ, which does not require the presence of a homologous template, is the predominant repair pathway for DSBs produced by ionizing radiation, and DNA-PK plays a central role in regulating the NHEJ of DSBs in the course of radiation therapy (23). DNA-PK is a trimeric protein consisting of a heterodimer formed between ku70 and ku880, which is recruited to the break first, and activates the catalytic subunit, DNA-PKcs (24). DNA-PKcs is a serine/threonine protein kinase belonging to the phosphoinositide 3-kinase-like family of protein kinase (PIKK) (20). Numerous studies have shown that it undergoes a series of phosphorylations in response to DSBs at the clusters of ABCDE (6 sites between Thr2609 and Thr2647) and PQR ( 5 sites between residues 2023 and 2056) (25). In order to investigate how sorafenib suppressed the repair of radiationinduced DSBs, we studied the effects of sorafenib on activated DNA-PK. We found that the level of activated DNA-PK was slightly reduced by combined sorafenib and radiation treatment, in a time-dependent manner.

In addition to its action as a direct radiosensitizer, sorafenib may also reduce tumor cell invasion by blocking MMP-9 production through the inhibition of the Raf-MAPK pathway, which was previously shown to induce the production of matrix metalloproteinases (26). MMP-9 is known to enhance the invasion of tumor cells through the controlled degradation of the extra-cellular matrix in a range of tumor types $(27,28)$. Our findings suggest that sorafenib also blocks MMP-9 in colon cancer cells, an effect that is enhanced by concomitant radiation. In addition to MMP-9, VEGF, which mediates angiogenesis via the activation of the PI3K/Akt and MAPK signaling cascade, has also been identified as an important target of sorafenib (5). We observed that, although phosphorylated VEGF2 was elevated as a result of irradiation alone, it was significantly downregulated by both combined sorafenib and radiation treatment. ERK is a key downstream component of the RAF/MEK/ERK signaling pathway and aberrant signaling through the ERK pathway could promote cell immortalization, proliferation and resistance to radiation (29). Western blot analysis demonstrated that radiation could result in the phosphorylation and hence activation of ERK, but that this was also suppressed by post-irradiation treatment with sorafenib. This was supported by our additional observation that VEGF treatment of endothelial cells significantly enhanced tube formation, and this too was blocked by sorafenib combined with radiation.

In summary, this study demonstrated that sorafenib can radiosensitize colon cancer cells through the inhibition of tumor cell survival, cell cycle regulation, DNA repair activity, tumor cell invasiveness and angiogenesis. These findings provide molecular evidence for the use of chemoradiation to treat colon cancer, and in vivo modeling should be used to further assess its suitability for clinical applications. The interaction between sorafenib and radiation in normal colorectal cells for normal tissue toxicity and complication requires further study. Furthermore, as the sensitizing effect of sorafenib in photon beam treatment is well characterized, it is important to compare its sensitizing effect and underlying mechanism for carbon beams in high LET, as well as other types of radiation in clinical applications to enhance the efficacy and safety of these forms of radiotherapy.

\section{Acknowledgements}

This study was supported by the National Research Foundation of Korea (NRF) grant funded by the Korea government (MSIP) (2014029534). 


\section{References}

1. Jemal A, Siegel R, Ward E, et al: Cancer statistics, 2008. CA Cancer J Clin 58: 71-96, 2008.

2. Saltz LB and Minsky B: Adjuvant therapy of cancers of the colon and rectum. Surg Clin North Am 82: 1035-1058, 2002.

3. Krook JE, Moertel CG, Gunderson LL, et al: Effective surgical adjuvant therapy for high-risk rectal carcinoma. N Engl J Med 324: 709-715, 1991.

4. Seiwert TY, Salama JK and Vokes EE: The concurrent chemoradiation paradigm - general principles. Nat Clin Pract Oncol 4: 86-100, 2007.

5. Wilhelm SM, Carter C, Tang L, et al: BAY 43-9006 exhibits broad spectrum oral antitumor activity and targets the RAF/ MEK/ERK pathway and receptor tyrosine kinases involved in tumor progression and angiogenesis. Cancer Res 64: 7099-7109, 2004.

6. Wilhelm SM, Adnane L, Newell P, Villanueva A, Llovet JM and Lynch M: Preclinical overview of sorafenib, a multikinase inhibitor that targets both Raf and VEGF and PDGF receptor tyrosine kinase signaling. Mol Cancer Ther 7: 3129-3140, 2008.

7. Iyer R, Fetterly G, Lugade A and Thanavala Y: Sorafenib: a clinical and pharmacologic review. Expert Opin Pharmacother 11: 1943-1955, 2010.

8. Siegel AB, Olsen SK, Magun A and Brown RS Jr: Sorafenib: where do we go from here? Hepatology 52: 360-369, 2010.

9. Escudier B ET, Stadler WM, Szczylik C, Oudard S, Siebels M, Negrier S, Chevreau C, Solska E, Desai AA, Rolland F, Demkow T, Hutson TE, Gore M, Freeman S, Schwartz B, Shan M, Simantov R, Bukowski RM and TARGET Study Group. Sorafenib in advanced clear-cell renal-cell carcinoma. New Engl J Med 356: 125-134, 2007.

10. Gupta-Abramson V, Troxel AB, Nellore A, et al: Phase II trial of sorafenib in advanced thyroid cancer. J Clin Oncol 26: 4714-4719, 2008.

11. Takimoto $\mathrm{CH}$ and Awada A: Safety and anti-tumor activity of sorafenib (Nexavar) in combination with other anti-cancer agents: a review of clinical trials. Cancer Chemother Pharmacol 61: 535-548, 2008

12. Kumar P, Benedict R, Urzua F, Fischbach C, Mooney D and Polverini P: Combination treatment significantly enhances the efficacy of antitumor therapy by preferentially targeting angiogenesis. Lab Invest 85: 756-767, 2005.

13. Plastaras JP, Kim SH, Liu YY, et al: Cell cycle-dependent and schedule-dependent antitumor effects of sorafenib combined with radiation. Cancer Res 67: 9443-9454, 2007.

14. Dal Lago L, D'Hondt V and Awada A: Selected combination therapy with sorafenib: a review of clinical data and perspectives in advanced solid tumors. Oncologist 13: 845-858, 2008.
15. Azad N, Annunziata C, Barrett T, et al: Dual targeting of vascular endothelial growth factor (VEGF) with sorafenib and bevacizumab: clinical and translational results. J Clin Oncol (Meeting abstracts) 25: 3542, 2007.

16. Kasibhatla M, Steinberg P, Meyer J, Ernstoff MS and George DJ: Radiation therapy and sorafenib: clinical data and rationale for the combination in metastatic renal cell carcinoma. Clin Genitourin Cancer 5: 291-294, 2007.

17. Horgan AM, Dawson LA, Swaminath A and Knox JJ: Sorafenib and radiation therapy for the treatment of advanced hepatocellular carcinoma. J Gastrointest Cancer 43: 344-348, 2012.

18. Jeong YK, Kim MS, Lee JY, et al: Sorafenib acts synergistically in combination with radiotherapy without causing intestinal damage in colorectal cancer. Tumori 99: 176-182, 2013.

19. Jackson SP: Sensing and repairing DNA double-strand breaks. Carcinogenesis 23: 687-696, 2002.

20. Rogakou EP, Pilch DR, Orr AH, Ivanova VS and Bonner WM: DNA double-stranded breaks induce histone $\mathrm{H} 2 \mathrm{AX}$ phosphorylation on serine 139. J Biol Chem 273: 5858-5868, 1998.

21. Bonner WM, Redon CE, Dickey JS, et al: GammaH2AX and cancer. Nat Rev Cancer 8: 957-967, 2008.

22. Bourton EC, Plowman PN, Smith D, Arlett CF and Parris CN: Prolonged expression of the $\gamma-\mathrm{H} 2 \mathrm{AX}$ DNA repair biomarker correlates with excess acute and chronic toxicity from radiotherapy treatment. Int J Cancer 129: 2928-2934, 2011.

23. Collis SJ, DeWeese TL, Jeggo PA and Parker AR: The life and death of DNA-PK. Oncogene 24: 949-961, 2005.

24. Smith GC and Jackson SP: The DNA-dependent protein kinase. Genes Dev 13: 916-934, 1999.

25. Douglas P, Sapkota G, Morrice N, et al: Identification of in vitro and in vivo phosphorylation sites in the catalytic subunit of the DNA-dependent protein kinase. Biochem J 368: 243-251, 2002.

26. Pignochino Y, Grignani G, Cavalloni G, et al: Sorafenib blocks tumour growth, angiogenesis and metastatic potential in preclinical models of osteosarcoma through a mechanism potentially involving the inhibition of ERK1/2, MCL-1 and ezrin pathways. Mol Cancer 8: 118, 2009.

27. Basset P, Okada A, Chenard MP, et al: Matrix metalloproteinases as stromal effectors of human carcinoma progression: therapeutic implications. Matrix Biol 15: 535-541, 1997.

28. Johnsen M, Lund LR, Rømer J, Almholt K and Dan $\varnothing$ K: Cancer invasion and tissue remodeling: common themes in proteolytic matrix degradation. Curr Opin Cell Biol 10: 667-671, 1998.

29. Roberts PJ and Der CJ: Targeting the Raf-MEK-ERK mitogenactivated protein kinase cascade for the treatment of cancer. Oncogene 26: 3291-3310, 2007. 